\title{
EDITORIAL
}

\section{HPLSE editorial tribute to Professor David Neely}

\author{
Colin Danson ${ }^{1,2,3}$ and Paul McKenna ${ }^{4}$ \\ ${ }^{1}$ High Power Laser Science and Engineering, Cambridge University Press, Cambridge, UK \\ ${ }^{2}$ AWE, Aldermaston, Reading, Berkshire, UK \\ ${ }^{3}$ CIFS, Blackett Laboratory, Imperial College London, London, UK \\ ${ }^{4}$ Department of Physics, SUPA, University of Strathclyde, Glasgow, UK \\ (Received 16 December 2020; accepted 21 December 2020)
}

\begin{abstract}
David Neely was an internationally recognised scientist who formed collaborations and friendships across the world. His passion for his work always shone through. He always made time for early-career scientists and became a mentor and supervisor to many. He was an active Editorial Board Member of the international journal High Power Laser Science and Engineering. Sadly, David was taken from us much too early. In this Editorial we pay tribute to his work through his publications in the journal.
\end{abstract}

Keywords: Editorial; David Neely; tribute

\section{Introduction}

David Neely completed a BSc Honours degree in Physics at Queen's University Belfast (QUB). He undertook a finalyear undergraduate project on flashlamp pumped lasers with Prof. Ciaran Lewis in 1986, subsequently starting a $\mathrm{PhD}$ in 1987 at QUB under Ciaran on the generation of soft X-ray lasers. David's PhD studies culminated in 1991 with the use of two opposed slab targets to compensate for refraction and increase the gain length product, enabling saturation using neon-like germanium at $23 \mathrm{~nm}$ with Vulcan at the Central Laser Facility (CLF). While writing up his $\mathrm{PhD}$ thesis he was employed as a Research Assistant at QUB in teaching and supporting the undergraduate research laboratories. In 1993 David moved to the Rutherford Appleton Laboratory to take up a position on the Vulcan laser and in 2005 took charge of the CLF's Experimental Science Group. He was Head of High Power Laser Science from 2010 to 2012 and was awarded a Science and Technology Facilities Council (STFC) Research Fellowship in 2012.

David naturally built up an incredible network of colleagues and friends throughout the world. He carried out experiments on lasers throughout Europe, Asia and the USA, contributing to experiments at the Lawrence

Correspondence to: C. Danson, CIFS, Blackett Laboratory, Imperial College London, London SW7 2AZ, UK. Email: c.danson@imperial.ac.uk
Livermore National Laboratory and the Brookhaven National Laboratory, for example. He held a Visiting Mitsuyuki Abe Chair position at the Proton Medical Research Center, Japan Atomic Energy Agency, giving him an even greater international profile.

He was appointed a Visiting Professor at the University of Strathclyde from 2008 to 2020 and worked very closely with Prof. Paul McKenna. This enabled him to co-supervise $\mathrm{PhD}$ students and enhance his research portfolio. He formed close relationships with all his students, which would be lasting, well after they left to take up jobs elsewhere, many at national and international laser facilities.

David also took a very active role on the Editorial Board of High Power Laser Science and Engineering (HPLSE). The journal hosted a biennial symposium in Suzhou, China, which he regularly attended, giving him an opportunity to consolidate his research collaborations in China and present his work in the international arena (Figure 1). He formed a very close relationship with two groups in China: Prof. Yutong Li at the Institute of Physics (IOP) in Beijing and Prof. Jie Zhang at Shanghai Jiao Tong University. This resulted in a government-funded UK-China collaboration, which allowed him not only to carry out work in China but also to bring students over to the UK. Because of his contribution to this work he was awarded the Medal of International Collaboration of the Chinese Academy of Sciences in 2017. 


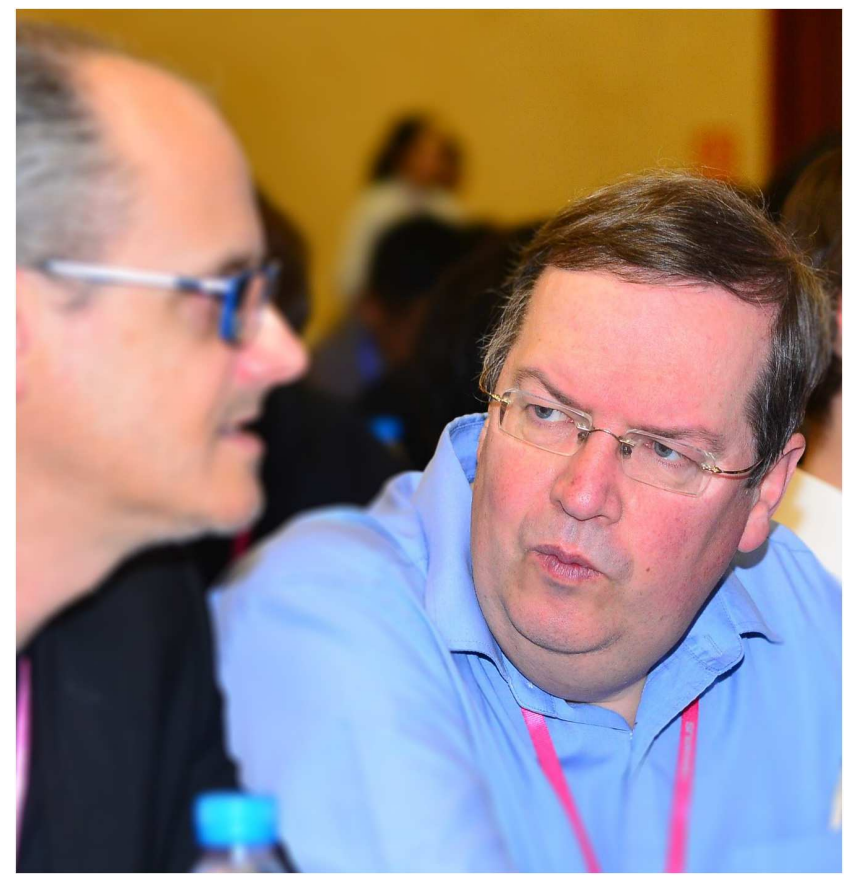

Figure 1. David Neely (right) in discussion with Leonida Gizzi (Istituto Nazionale di Ottica, Pisa, Italy) at the HPLSE symposium, Suzhou, China, 2018.

In the weeks before his untimely death on 27 August 2020, David was working on his contribution to a manuscript on the history of high-power laser research and development in the UK, for publication in HPLSE ${ }^{[1]}$. This was one of nearly 400 publications that David co-authored throughout his career. His work was mainly in the field of highpower laser-plasma interactions and the applications of the resulting beams of energetic particles and radiation, and he published extensively in this area. In striving to develop the field, he worked on new instrumentation (including detectors and diagnostics) and methodologies (e.g., plasma optics and targetry). As a result, David has published a rich body of high-quality work across a broad range of topics linked to the field.

This Editorial is in memory of David and his scientific achievements. We have brought together the paper that he was working on at the time of his death and other papers that he published in the journal.

\section{Scientific highlights through publications in HPLSE}

The first three of David's HPLSE papers that we highlight provide an overview of ultra-high-power laser systems and laser science. This trio of papers began in 2014 with a publication on pulse fidelity in petawatt-class laser systems $^{[2]}$. The paper summarized the development on the UK's three ultra-high-power laser facilities. These facilities represent the two main classes of petawatt facilities: the mixed OPCPA/Nd:glass high-energy systems of Orion at the
Atomic Weapons Establishment; and Vulcan and the ultrashort-pulse Ti:sapphire system of Astra Gemini, both at the CLF at the STFC's Rutherford Appleton Laboratory. Many of the techniques used to enhance and control the pulse generation and delivery to target of ultra-high-power pulses have been pioneered on these facilities.

In compiling the paper, an annex was intended to summarize the current status of ultra-high-power lasers throughout the world. As this annex grew, it became significantly longer than the paper itself and so the decision was made to split this out and produce a separate comprehensive review, 'Petawatt Class Lasers Worldwide', which was published in $2015^{[3]}$. This has become an important review paper for researchers in the field and has attracted over 300 citations to date. These include citation by the Nobel Committee in Physics in its 2018 scientific background paper 'Groundbreaking Inventions in Laser Physics ${ }^{[4]}$ for the award to Profs. Donna Strickland and Gerard Mourou for the invention of the technique of chirped pulse amplification ${ }^{[5]}$, which was shared with Prof. Arthur Ashkin for his work on optical tweezers and their application to biological systems.

As testimony to the rapid growth of the field of petawatt laser-plasma science, this paper was followed in 2019 with a presentation of petawatt- and exawatt-class lasers worldwide $^{[6]}$. This review paper built on the earlier review paper 'Petawatt Class Lasers Worldwide' but was much more comprehensive, providing a discussion of the history of ultrahigh-power lasers and a look forward to which techniques and research activities were emerging to produce the next generation of lasers.

In parallel with his interests in developing high-power lasers, David made leading contributions to a large body of work on various aspects of the interaction of high-power laser pulses with gaseous and solid density plasma. He introduced a number of novel ideas to the development of laser-driven ion acceleration and contributed to studies that used ion acceleration to diagnose the physics of fast electron generation and transport in dense plasma, which is directly relevant to the development of advanced ignition schemes for inertial fusion. An example of the latter is that the investigation into the effect of the electronic sheath field, responsible for ion acceleration, on fast electron dynamics within foil targets is reported in Ref. [7]. In that paper, it is shown that energetic electrons that do not overcome the electrostatic potential formed at the target rear are reflected and reflux within the foil. The temporal characteristics of this population of refluxing electrons and those that escape the target foil are investigated and correlated with the magnitude and spatial and temporal evolution of the electrostatic fields. This work is one of many that David led on this topic.

David is well known for introducing new innovations into the field of laser-plasma science and one such innovation was the use of ultrathin foil targets for ion acceleration. This was a direct result of David challenging the Target 
Fabrication group at the CLF to produce the thinnest foils possible to enhance the electrostatic fields responsible for ion acceleration at the rear of the foil. In addition to investigating ion acceleration from ultrathin foils, David contributed to numerous recent studies into laser pulse propagation and collective electron dynamics in ultrathin foils undergoing expansion and becoming relativistically transparent to the intense laser light. In an example of this work, Ref. [8] reports on an experimental and numerical investigation into the collective response of electrons in an ultrathin foil target irradiated by an ultraintense laser pulse. It is shown that when the target thickness is decreased such that it becomes relativistically transparent to the laser light early in the interaction, diffraction of the transmitted laser light occurs through a so-called 'relativistic plasma aperture', inducing structure in the spatial-intensity profile of the beam of accelerated electrons. It is also shown that the electron beam profile can be modified by variation of the target thickness and degree of ellipticity in the laser polarization.

A related study ${ }^{[9]}$ reports the role of magnetic field evolution in filamentary structures that form within the beam of protons accelerated during the interaction of an intense laser pulse with an ultrathin foil target. Such behaviour is shown to be dependent on the formation time of quasi-static magnetic field structures throughout the target volume and the extent of the rear surface proton expansion over the same period. By controlling the intensity profile of the laser drive, via the use of two temporally separated pulses, both the initial rear surface proton expansion and magnetic field formation time can be varied, resulting in modification to the degree of filamentary structure present within the laser-driven proton beam. In separate work, David used dual laser pulses to drive proton acceleration and demonstrated enhanced energy transfer using this approach.

David's other work on novel targets includes mass-limited targets to limit lateral energy loss in thin foils and structured targets to enhance laser energy absorption by electrons or to generate point-like sources of energetic radiation. He contributed to the study published in Ref. [10], in which diffraction and speckle patterns measured in the spatialintensity profile of laser light reflected during the interaction with a microstructured target are investigated experimentally, and the potential to apply this as a diagnostic of laser focus and plasma temperature is explored. Using modelling, the possibility of applying this as a diagnostic of the evolving plasma surface is discussed. In another example involving innovative targets, David led a study ${ }^{[1]}$ into the use of constrained targets to enhance laser-driven Bremsstrahlung emission for industrial radiography applications. By limiting the lateral dimensions of the target, $\mathrm{X}$-ray generation was confined to a localized region, enabling imaging with enhanced resolution and contrast, compared to the use of unconstrained foil targets. The sheath field formed around the constrained target was also shown to enhance electron refluxing within the target, driving a brighter source of $\mathrm{X}$ rays. David was keen to develop novel laser-driven X-ray sources for applications such as non-destructive imaging.

In keeping with the theme of secondary radiation generation, David also made important contributions to the development of terahertz $(\mathrm{THz})$ radiation sources driven by intense laser-solid interactions. He published two papers on this topic in $\operatorname{HPLSE}^{[12,13]}$. In an early work in $2014^{[10]}$, the effects of target pre-heating and expansion on $\mathrm{THz}$ radiation production from intense laser-solid interactions were first explored. The total energy of the $\mathrm{THz}$ radiation is found to decrease by approximately a factor of two when preheating the target compared to a cold target reference, and is attributed to an increase in the scale length of the preformed plasma in the region in which the $\mathrm{THz}$ radiation is generated. The results show the importance of controlling the pre-plasma scale length for $\mathrm{THz}$ production, and this is a theme that is reflected in other work that David contributed to on the role of pre-plasma in ion acceleration. In related work on $\mathrm{THz}$ generation, published in $2019^{[13]}$, backwarddirected $\mathrm{THz}$ radiation from intense picosecond laser-solid interactions was investigated, using a multichannel calorimeter system jointly introduced by David. The paper reports on the dependency of $\mathrm{THz}$ energy and spectrum on the drive laser pulse energy, target thickness and pre-plasma scale length. It shows that a large-scale pre-plasma can enhance the high-frequency component backward-directed $\mathrm{THz}$ radiation.

Those who knew David understood his passion for solving challenging problems that limited progress in experimental science. One such issue is that of laser-generated electromagnetic pulses (EMP), which can damage electronics, particularly in detectors and computers positioned near the target interaction chamber. EMP emission is caused by the acceleration of hot electrons inside the target, which produces radiation across a wide band from $\mathrm{DC}$ to $\mathrm{THz}$ frequencies. In 2018, David published a paper ${ }^{[14]}$, involving researchers from five institutions, in which it was demonstrated experimentally how EMP can be readily and effectively reduced. Using novel targets, many of which were introduced by David, it was demonstrated that target stalk geometry, material composition, geodesic path length and foil surface area can all play a significant role in the reduction of EMP. A combination of electromagnetic wave and 3D particle-in-cell simulations helped in understanding the effects of stalk geometry on EMP, providing an opportunity for comparison with existing charge separation models. This work is a significant achievement and one in which David had a leading role.

In a follow-up study, published in $2020^{[15]}$, David brought together a collaboration of researchers from 21 institutions across Europe and China to review EMP generation, detection and mitigation in the interaction of high-power, high-energy laser pulses with different types of solid targets. 
The resulting review paper includes new experimental data obtained independently at several international laboratories and analyses the mechanisms of electromagnetic field generation as a function of the intensity and the spectral range of emissions they produce. The work focuses on the $\mathrm{GHz}$ frequency domain, which is the most damaging for electronics, but also discusses the physics of electromagnetic emissions in other spectral domains, in particular $\mathrm{THz}$ and MHz. Theoretical models and numerical simulations are compared with the results of experimental measurements to provide new insight into the underlying physical processes and provide a basis for developing techniques to mitigate the EMP threat and to harness electromagnetic emissions. This review paper provides an important reference work on EMP in laser-plasma interactions and in itself reflects David's legacy in the field.

David was keen to push the boundaries of what is possible in laser-plasma science. He strongly promoted the development of multi-petawatt lasers and the new topics that these would enable. In preparation for future experiments on the next generation of high-power laser facilities, David contributed to a number of numerical studies into the new physics that may be enabled. Ref. [16] provides an example work in which approaches involving colliding laser pulses on existing petawatt laser facilities are discussed as a route to providing insight into the strong-field quantum electrodynamics (QED) physics that may be explored at multi-petawatt-scale facilities. This includes radiation reaction physics and Breit-Wheeler pair production. David's untimely passing means that he will not see this new chapter in high-power laser-plasma science. His legacy is that many of the innovations he has introduced in the field will be applied to enable experiments in this new-intensity regime, and many of the scientists he helped to train will be performing those experiments.

\section{Summary}

David was much admired and respected. He worked tirelessly to develop high-power laser-plasma science and to educate new scientists in the field. His passion and enthusiasm for his science and for mentoring younger scientists was infectious. He will be deeply missed by many in the community. As a member of the Editorial Board for HPLSE, we are sure that David would be pleased to see this Editorial with a summary of the works he published in it.

\section{Acknowledgements}

The authors would like to thank David's family, in particular his wife, Lesley, for helping to put this tribute together.

\section{Appendix - abstracts of the papers published by Prof. David Neely in HPLSE}

Effects of target pre-heating and expansion on terahertz radiation production from intense laser-solid interactions

X. H. Yuan, Y. Fang, D. C. Carroll, D. A. MacLellan, F. Du, N. Booth, M. Burza, M. Chen, R. J. Gray, Y. F. Jin, Y. T. Li, Y. Liu, D. Neely, H. Powell, G. Scott, C.-G. Wahlström, J. Zhang, P. McKenna, Z. M. Sheng

High Power Laser Sci. Eng. 2, e5 (2014)

Abstract: The first experimental measurements of intense $\left(\sim 7 \times 10^{19} \mathrm{~W} \mathrm{~cm}^{-2}\right)$ laser-driven terahertz $(\mathrm{THz})$ radiation from a solid target which is preheated by an intense pulse of laser-accelerated protons is reported. The total energy of the $\mathrm{THz}$ radiation is found to decrease by approximately a factor of 2 compared to a cold target reference. This is attributed to an increase in the scale length of the preformed plasma, driven by proton heating, at the front surface of the target, where the $\mathrm{THz}$ radiation is generated. The results show the importance of controlling the preplasma scale length for $\mathrm{THz}$ production.

\section{Pulse fidelity in ultra-high-power (petawatt class) laser systems}

Colin Danson, David Neely, David Hillier

High Power Laser Sci. Eng. 2, e34 (2014)

Abstract: There are several petawatt-scale laser facilities around the world and the fidelity of the pulses to target is critical in achieving the highest focused intensities and the highest possible contrast. The United Kingdom has three such laser facilities which are currently open for access to the academic community: Orion at AWE, Aldermaston and Vulcan \& Astra-Gemini at the Central Laser Facility (CLF), STFC (Science and Technology Facilities Council) Rutherford Appleton Laboratory (RAL). These facilities represent the two main classes of petawatt facilities: the mixed OPCPA/Nd:glass high-energy systems of Orion and Vulcan and the ultra-short-pulse Ti:Sapphire system of Astra-Gemini. Many of the techniques used to enhance and control the pulse generation and delivery to target have been pioneered on these facilities. In this paper, we present the system designs which make this possible and discuss the contrast enhancement schemes that have been implemented.

\section{Petawatt class lasers worldwide}

Colin Danson, David Hillier, Nicholas Hopps, David Neely 
High Power Laser Sci. Eng. 3, e3 (2015)

Abstract: The use of ultra-high intensity laser beams to achieve extreme material states in the laboratory has become almost routine with the development of the petawatt laser. Petawatt class lasers have been constructed for specific research activities, including particle acceleration, inertial confinement fusion and radiation therapy, and for secondary source generation (X-rays, electrons, protons, neutrons and ions). They are also now routinely coupled, and synchronized, to other large scale facilities including megajoule scale lasers, ion and electron accelerators, Xray sources and z-pinches. The authors of this paper have tried to compile a comprehensive overview of the current status of petawatt class lasers worldwide. The definition of 'petawatt class' in this context is a laser that delivers $>200$ TW.

Influence of laser polarization on collective electron dynamics in ultraintense laser-foil interactions

Bruno Gonzalez-Izquierdo, Ross J. Gray, Martin King, Robbie Wilson, Rachel J. Dance, Haydn Powell, David A. MacLellan, John McCreadie, Nicholas M. H. Butler, Steve Hawkes, James S. Green, Chris D. Murphy, Luca C. Stockhausen, David C. Carroll, Nicola Booth, Graeme G. Scott, Marco Borghesi, David Neely, Paul McKenna

\section{High Power Laser Sci. Eng. 4, e33 (2016)}

Abstract: The collective response of electrons in an ultrathin foil target irradiated by an ultraintense $(\sim 6 \times$ $10^{20} \mathrm{~W} \mathrm{~cm} \mathrm{~cm}^{-2}$ ) laser pulse is investigated experimentally and via $3 \mathrm{D}$ particle-in-cell simulations. It is shown that if the target is sufficiently thin that the laser induces significant radiation pressure, but not thin enough to become relativistically transparent to the laser light, the resulting relativistic electron beam is elliptical, with the major axis of the ellipse directed along the laser polarization axis. When the target thickness is decreased such that it becomes relativistically transparent early in the interaction with the laser pulse, diffraction of the transmitted laser light occurs through a so called 'relativistic plasma aperture', inducing structure in the spatial-intensity profile of the beam of energetic electrons. It is shown that the electron beam profile can be modified by variation of the target thickness and degree of ellipticity in the laser polarization.

\section{EMP control and characterization on high-power laser systems}

P. Bradford, N. C. Woolsey, G. G. Scott, G. Liao, H. Liu, Y. Zhang, B. Zhu, C. Armstrong, S. Astbury, C. Brenner, P. Brummitt, F. Consoli, I. East, R. Gray, D. Haddock,
P. Huggard, P. J. R. Jones, E. Montgomery, I. Musgrave, P. Oliveira, D. R. Rusby, C. Spindloe, B. Summers, E. Zemaityte, Z. Zhang, Y. Li, P. McKenna, D. Neely

High Power Laser Sci. Eng. 6, e21 (2018), Editors' Pick

Abstract: Giant electromagnetic pulses (EMP) generated during the interaction of high-power lasers with solid targets can seriously degrade electrical measurements and equipment. EMP emission is caused by the acceleration of hot electrons inside the target, which produce radiation across a wide band from DC to terahertz frequencies. Improved understanding and control of EMP is vital as we enter a new era of high repetition rate, high intensity lasers (e.g. the Extreme Light Infrastructure). We present recent data from the Vulcan laser facility that demonstrates how EMP can be readily and effectively reduced. Characterization of the EMP was achieved using B-dot and D-dot probes that took measurements for a range of different target and laser parameters. We demonstrate that target stalk geometry, material composition, geodesic path length and foil surface area can all play a significant role in the reduction of EMP. A combination of electromagnetic wave and 3D particle-in-cell simulations is used to inform our conclusions about the effects of stalk geometry on EMP, providing an opportunity for comparison with existing charge separation models.

Reflection of intense laser light from microstructured targets as a potential diagnostic of laser focus and plasma temperature

J. Jarrett, M. King, R. J. Gray, N. Neumann, L. Döhl, C. D. Baird, T. Ebert, M. Hesse, A. Tebartz, D. R. Rusby, N. C. Woolsey, D. Neely, M. Roth, P. McKenna

High Power Laser Sci. Eng. 7, e2 (2019)

Abstract: The spatial-intensity profile of light reflected during the interaction of an intense laser pulse with a microstructured target is investigated experimentally and the potential to apply this as a diagnostic of the interaction physics is explored numerically. Diffraction and speckle patterns are measured in the specularly reflected light in the cases of targets with regular groove and needle-like structures, respectively, highlighting the potential to use this as a diagnostic of the evolving plasma surface. It is shown, via ray-tracing and numerical modelling, that for a laser focal spot diameter smaller than the periodicity of the target structure, the reflected light patterns can potentially be used to diagnose the degree of plasma expansion, and by extension the local plasma temperature, at the focus of the intense laser light. The reflected patterns could also be used to diagnose the size of the laser focal spot during a high-intensity interaction when using a regular structure with known spacing. 
Study of backward terahertz radiation from intense picosecond laser-solid interactions using a multichannel calorimeter system

H. Liu, G.-Q. Liao, Y.-H. Zhang, B.-J. Zhu, Z. Zhang, Y.-T. Li, G. G. Scott, D. Rusby, C. Armstrong, E. Zemaityte, P. Bradford, N. Woolsey, P. Huggard, P. McKenna, D. Neely

High Power Laser Sci. Eng. 7, e6 (2019)

Abstract: A multichannel calorimeter system is designed and constructed which is capable of delivering single-shot and broad-band spectral measurement of terahertz $(\mathrm{THz})$ radiation generated in intense laser-plasma interactions. The generation mechanism of backward $\mathrm{THz}$ radiation (BTR) is studied by using the multichannel calorimeter system in an intense picosecond laser-solid interaction experiment. The dependence of the BTR energy and spectrum on laser energy, target thickness and pre-plasma scale length is obtained. These results indicate that coherent transition radiation is responsible for the low-frequency component $(<1 \mathrm{THz})$ of BTR. It is also observed that a large-scale preplasma primarily enhances the high-frequency component $(>3 \mathrm{THz})$ of BTR.

Quantum electrodynamics experiments with colliding petawatt laser pulses

I. C. E. Turcu, B. Shen, D. Neely, G. Sarri, K. A. Tanaka, P. McKenna, S. P. D. Mangles, T.-P. Yu, W. Luo, X.-L. Zhu, Y. Yin

High Power Laser Sci. Eng. 7, e10 (2019)

Abstract: A new generation of high power laser facilities will provide laser pulses with extremely high powers of 10 petawatt $(\mathrm{PW})$ and even $100 \mathrm{PW}$, capable of reaching intensities of $10^{23} \mathrm{~W} / \mathrm{cm}^{2}$ in the laser focus. These ultrahigh intensities are nevertheless lower than the Schwinger intensity $I_{S}=2.3 \times 10^{29} \mathrm{~W} / \mathrm{cm}^{2}$ at which the theory of quantum electrodynamics (QED) predicts that a large part of the energy of the laser photons will be transformed to hard Gamma-ray photons and even to matter, via electronpositron pair production. To enable the investigation of this physics at the intensities achievable with the next generation of high power laser facilities, an approach involving the interaction of two colliding PW laser pulses is being adopted. Theoretical simulations predict strong QED effects with colliding laser pulses of $\geqslant 10 \mathrm{PW}$ focused to intensities $\geqslant 10^{22} \mathrm{~W} / \mathrm{cm}^{2}$.

Role of magnetic field evolution on filamentary structure formation in intense laser-foil interactions

M. King, N. M. H. Butler, R. Wilson, R. Capdessus, R. J. Gray, H. W. Powell, R. J. Dance, H. Padda, B. GonzalezIzquierdo, D. R. Rusby, N. P. Dover, G. S. Hicks, O. C.
Ettlinger, C. Scullion, D. C. Carroll, Z. Najmudin, M. Borghesi, D. Neely, P. McKenna

High Power Laser Sci. Eng. 7, e14 (2019)

Abstract: Filamentary structures can form within the beam of protons accelerated during the interaction of an intense laser pulse with an ultrathin foil target. Such behaviour is shown to be dependent upon the formation time of quasi-static magnetic field structures throughout the target volume and the extent of the rear surface proton expansion over the same period. This is observed via both numerical and experimental investigations. By controlling the intensity profile of the laser drive, via the use of two temporally separated pulses, both the initial rear surface proton expansion and magnetic field formation time can be varied, resulting in modification to the degree of filamentary structure present within the laser-driven proton beam.

Bremsstrahlung emission from high power laser interactions with constrained targets for industrial radiography

C. D. Armstrong, C. M. Brenner, C. Jones, D. R. Rusby, Z. E. Davidson, Y. Zhang, J. Wragg, S. Richards, C. Spindloe, P. Oliveira, M. Notley, R. Clarke, S. R. Mirfayzi, S. Kar, Y. Li, T. Scott, P. McKenna, D. Neely

High Power Laser Sci. Eng. 7, e24 (2019), Editors' Pick

Abstract: Laser-solid interactions are highly suited as a potential source of high energy X-rays for nondestructive imaging. A bright, energetic X-ray pulse can be driven from a small source, making it ideal for high resolution X-ray radiography. By limiting the lateral dimensions of the target we are able to confine the region over which X-rays are produced, enabling imaging with enhanced resolution and contrast. Using constrained targets we demonstrate experimentally a (20 \pm 3$) \mu \mathrm{m} X$-ray source, improving the image quality compared to unconstrained foil targets. Modelling demonstrates that a larger sheath field envelope around the perimeter of the constrained targets increases the proportion of electron current that recirculates through the target, driving a brighter source of X-rays.

Effect of rear surface fields on hot, refluxing and escaping electron populations via numerical simulations

D. R. Rusby, C. D. Armstrong, G. G. Scott, M. King, P. McKenna, D. Neely

High Power Laser Sci. Eng. 7, e45 (2019)

Abstract: After a population of laser-driven hot electrons traverses a limited thickness solid target, these electrons will encounter the rear surface, creating $\mathrm{TV} / \mathrm{m}$ fields that heavily influence the subsequent hot-electron propagation. Electrons that fail to overcome the electrostatic potential reflux back into the target. Those electrons that do overcome the field will escape the target. Here, using the particle- 
in-cell (PIC) code EPOCH and particle tracking of a large population of macro-particles, we investigate the refluxing and escaping electron populations, as well as the magnitude, spatial and temporal evolution of the rear surface electrostatic fields. The temperature of both the escaping and refluxing electrons is reduced by $30 \%-50 \%$ when compared to the initial hot-electron temperature as a function of intensity between 1019 and $1021 \mathrm{~W} / \mathrm{cm}^{2}$. Using particle tracking we conclude that the highest energy internal hot electrons are guaranteed to escape up to a threshold energy, below which only a small fraction are able to escape the target. We also examine the temporal characteristic of energy changes of the refluxing and escaping electrons and show that the majority of the energy change is as a result of the temporally evolving electric field that forms on the rear surface.

\section{Petawatt and exawatt class lasers worldwide}

Colin N. Danson, Constantin Haefner, Jake Bromage, Thomas Butcher, Jean-Christophe F. Chanteloup, Enam A. Chowdhury, Almantas Galvanauskas, Leonida A. Gizzi, Joachim Hein, David I. Hillier, Nicholas W. Hopps, Yoshiaki Kato, Efim A. Khazanov, Ryosuke Kodama, Georg Korn, Ruxin Li, Yutong Li, Jens Limpert, Jingui Ma, Chang Hee Nam, David Neely, Dimitrios Papadopoulos, Rory R. Penman, Liejia Qian, Jorge J. Rocca, Andrey A. Shaykin, Craig W. Siders, Christopher Spindloe, Sándor Szatmári, Raoul M. G. M. Trines, Jianqiang Zhu, Ping Zhu, Jonathan D. Zuegel

\section{High Power Laser Sci. Eng. 7, e54 (2019), Editors' Pick}

Abstract: In the 2015 review paper 'Petawatt Class Lasers Worldwide' a comprehensive overview of the current status of high-power facilities of $>200 \mathrm{TW}$ was presented. This was largely based on facility specifications, with some description of their uses, for instance in fundamental ultrahigh-intensity interactions, secondary source generation, and inertial confinement fusion (ICF). With the 2018 Nobel Prize in Physics being awarded to Professors Donna Strickland and Gerard Mourou for the development of the technique of chirped pulse amplification (CPA), which made these lasers possible, we celebrate by providing a comprehensive update of the current status of ultra-high-power lasers and demonstrate how the technology has developed. We are now in the era of multi-petawatt facilities coming online, with 100 PW lasers being proposed and even under construction. In addition to this there is a pull towards development of industrial and multi-disciplinary applications, which demands much higher repetition rates, delivering high-average powers with higher efficiencies and the use of alternative wavelengths: mid-IR facilities. So apart from a comprehensive update of the current global status, we want to look at what technologies are to be deployed to get to these new regimes, and some of the critical issues facing their development.

\section{Laser produced electromagnetic pulses: generation, detection and mitigation}

Fabrizio Consoli, Vladimir T. Tikhonchuk, Matthieu Bardon, Philip Bradford, David C. Carroll, Jakub Cikhardt, Mattia Cipriani, Robert J. Clarke, Thomas E. Cowan, Colin N. Danson, Riccardo De Angelis, Massimo De Marco, JeanLuc Dubois, Bertrand Etchessahar, Alejandro Laso Garcia, David I. Hillier, Ales Honsa, Weiman Jiang, Viliam Kmetik, Josef Krása, Yutong Li, Frédéric Lubrano, Paul McKenna, Josefine Metzkes-Ng, Alexandre Poyé, Irene Prencipe, Piotr Rączka, Roland A. Smith, Roman Vrana, Nigel C. Woolsey, Egle Zemaityte, Yihang Zhang, Zhe Zhang, Bernhard Zielbauer, David Neely

High Power Laser Sci. Eng. 8, e22 (2020), Editors' Pick

Abstract: This paper provides an up-to-date review of the problems related to the generation, detection and mitigation of strong electromagnetic pulses created in the interaction of high-power, high-energy laser pulses with different types of solid targets. It includes new experimental data obtained independently at several international laboratories. The mechanisms of electromagnetic field generation are analyzed and considered as a function of the intensity and the spectral range of emissions they produce. The major emphasis is put on the $\mathrm{GHz}$ frequency domain, which is the most damaging for electronics and may have important applications. The physics of electromagnetic emissions in other spectral domains, in particular $\mathrm{THz}$ and $\mathrm{MHz}$, is also discussed. The theoretical models and numerical simulations are compared with the results of experimental measurements, with special attention to the methodology of measurements and complementary diagnostics. Understanding the underlying physical processes is the basis for developing techniques to mitigate the electromagnetic threat and to harness electromagnetic emissions, which may have promising applications.

\section{References}

1. C. Danson, M. White, J. Barr, T. Bett, P. Blyth, D. Bowley, C. Brenner, B. Collins, N. Croxford, B. Dangor, L. Devereux, P. E Dyer, A. Dymoke-Bradshaw, C. Edwards, P. Ewart, A. Ferguson, J. Girkin, D. Hall, D. Hanna, W. Harris, D. Hillier, C. Hooker, S. Hooker, N. Hopps, J. Hull, D. Hunt, D. Jaroszynski, M. Kempenaars, H. Kessler, S. P. Knight, S. Knight, A. Knowles, C. Lewis, K. Lipton, A. Littlechild, J. Littlechild, P. Maggs, G. M. OBE, S. Mangles, W. Martin, P. McKenna, R. Moore, C. Morrison, Z. Najmudin, D. Neely, G. New, M. Norman, T. Paine, A. Parker, R. Penman, G. Pert, C. Pietraszewski, A. Randewich, N. Rizvi, N. S. MBE, Z.-M. Sheng, D. Slater, R. Smith, C. Spindloe, R. Taylor, G. Thomas, J. Tisch, J. Wark, C. Webb, S. M. Wiggins, D. Willford, and T. Winstone, High Power Laser Sci. Eng. (to be published). 
2. C. Danson, D. Neely, and D. Hillier, High Power Laser Sci. Eng. 2, e34 (2014).

3. C. Danson, D. Hillier, N. Hopps, and D. Neely, High Power Laser Sci. Eng. 3, e3 (2015).

4. Nobel Prize Website, https://www.nobelprize.org/uploads/ 2018/10/advanced-physicsprize2018.pdf (2018).

5. D. Strickland and G. Mourou, Opt. Commun. 56, 219 (1985).

6. C. N. Danson, C. Haefner, J. Bromage, T. Butcher, J.-C. F. Chanteloup, E. A. Chowdhury, A. Galvanauskas, L. A. Gizzi, J. Hein, D. I. Hillier, N. W. Hopps, Y. Kato, E. A. Khazanov, R. Kodama, G. Korn, R. Li, Y. Li, J. Limpert, J. Ma, C. H. Nam, D. Neely, D. Papadopoulos, R. R. Penman, L. Qian, J. J. Rocca, A. A. Shaykin, C. W. Siders, C. Spindloe, S. Szatmári, R. M. G. M. Trines, J. Zhu, P. Zhu, and J. D. Zuegel, High Power Laser Sci. Eng. 7, e54 (2019).

7. D. R. Rusby, C. D. Armstrong, G. G. Scott, M. King, P. McKenna, and D. Neely, High Power Laser Sci. Eng. 7, e45 (2019).

8. B. Gonzalez-Izquierdo, R. J. Gray, M. King, R. Wilson, R. J. Dance, H. Powell, D. A. MacLellan, J. McCreadie, N. M. H. Butler, S. Hawkes, J. S. Green, C. D. Murphy, L. C. Stockhausen, D. C. Carroll, N. Booth, G. G. Scott, M. Borghesi, D. Neely, and P. McKenna, High Power Laser Sci. Eng. 4, e33 (2016).

9. M. King, N. M. H. Butler, R. Wilson, R. Capdessus, R. J. Gray, H. W. Powell, R. J. Dance, H. Padda, B. Gonzalez-Izquierdo, D. R. Rusby, N. P. Dover, G. S. Hicks, O. C. Ettlinger, C. Scullion, D. C. Carroll, Z. Najmudin, M. Borghesi, D. Neely, and P. McKenna, High Power Laser Sci. Eng. 7, e14 (2019).

10. J. Jarrett, M. King, R. J. Gray, N. Neumann, L. Döhl, C. D. Baird, T. Ebert, M. Hesse, A. Tebartz, D. R. Rusby, N. C. Woolsey, D. Neely, M. Roth, and P. McKenna, High Power Laser Sci. Eng. 7, e2 (2019).
11. C. D. Armstrong, C. M. Brenner, C. Jones, D. R. Rusby, Z. E. Davidson, Y. Zhang, J. Wragg, S. Richards, C. Spindloe, P. Oliveira, M. Notley, R. Clarke, S. R. Mirfayzi, S. Kar, Y. Li, T. Scott, P. McKenna, and D. Neely, High Power Laser Sci. Eng. 7, e24 (2019).

12. X. H. Yuan, Y. Fang, D. C. Carroll, D. A. MacLellan, F. Du, N. Booth, M. Burza, M. Chen, R. J. Gray, Y. F. Jin, Y. T. Li, Y. Liu, D. Neely, H. Powell, G. Scott, C.-G. Wahlström, J. Zhang, P. McKenna, and Z. M. Sheng, High Power Laser Sci. Eng. 2, e5 (2014).

13. H. Liu, G.-Q. Liao, Y.-H. Zhang, B.-J. Zhu, Z. Zhang, Y.T. Li, G. G. Scott, D. Rusby, C. Armstrong, E. Zemaityte, P. Bradford, N. Woolsey, P. Huggard, P. McKenna, and D. Neely, High Power Laser Sci. Eng. 7, e6 (2019).

14. P. Bradford, N. C. Woolsey, G. G. Scott, G. Liao, H. Liu, Y. Zhang, B. Zhu, C. Armstrong, S. Astbury, C. Brenner, P. Brummitt, F. Consoli, I. East, R. Gray, D. Haddock, P. Huggard, P. J. R. Jones, E. Montgomery, I. Musgrave, P. Oliveira, D. R. Rusby, C. Spindloe, B. Summers, E. Zemaityte, Z. Zhang, Y. Li, P. McKenna, and D. Neely, High Power Laser Sci. Eng. 6, e21 (2018).

15. F. Consoli, V. T. Tikhonchuk, M. Bardon, P. Bradford, D. C. Carroll, J. Cikhardt, M. Cipriani, R. J. Clarke, T. E. Cowan, C. N. Danson, R. De Angelis, M. De Marco, J.-L. Dubois, B. Etchessahar, A. L. Garcia, D. I. Hillier, A. Honsa, W. Jiang, V. Kmetik, J. Krása, Y. Li, F. Lubrano, P. McKenna, J. MetzkesNg, A. Poyé, I. Prencipe, P. Ra̧czka, R. A. Smith, R. Vrana, N. C. Woolsey, E. Zemaityte, Y. Zhang, Z. Zhang, B. Zielbauer, and D. Neely, High Power Laser Sci. Eng. 8, e22 (2020).

16. I. C. E. Turcu, B. Shen, D. Neely, G. Sarri, K. A. Tanaka, P. McKenna, S. P. D. Mangles, T.-P. Yu, W. Luo, X.L. Zhu, and Y. Yin, High Power Laser Sci. Eng. 7, e10 (2019). 